Z. klin. Chem. u. klin. Biochem.

7. Jg., S. 333-338, Juli 1969

\title{
Regulationsstörungen \\ bei akuter intermittierender Porphyrie und Bleivergiftung
}

\author{
Von L. GoreCzKY und I. Róth
}

Aus dem Zentrallaboratorium und der III. Mediziniscben Abteilung des MÁV-Krankenbauses, Budapest, Ungarn

(Eingegangen am 20. Dezember 1968)

Herrn Prof. Dr. Joacbin Brugsch zum 60. Geburtstag gewidmet

Es werden die Symptome der akuten intermittierenden Porphyrie und der Bleivergiftung verglichen; es konnten ähnliche Veränderungen beobachtet werden. Bei Störungen des Porphyrinstoffwechsels findet man Hinweise auf Enzyminduktion oder Enzymhemmung. In beiden Fällen aber kommt es zur Vermehrung von Metaboliten, die die Grundlage für die klinisch-chemische Diagnose bilden. Boi Bleivergiftung kommt es nahezu auf jeder Stufe der Hämsynthese zu Störungen. Zu wenig beachtet ist die von BrugsCr bei der Bleivergiftung beobachtete Vermehrung des Uroporphyrinogens. Dabei wird der Porphobilinogen-Wert im Harn normal gefunden. Uroporphyrinbildung beim Stehen des Harns ist nicht anzunehmen.

Mineral- und Wasserhaushalt sind bei beiden Krankheitsformen gestört. Dies wird auf eine gesteigerte Sekretion des antidiuretischen Hormons bezogen. Ebenso ist eine gesteigerte Absonderung des melanophoren-stimulierenden, des somatotropen und des thyreotropen Hormons der Hypophyse zu beobachten. Die erhöhte Hormonabgabe der Hypophyse ist auf eine anatomische Veränderung im Hypothalamus zurückzuführen, welche sowohl an Porphyrikern wie auch tierexperimentell nachweisbar ist.

\section{Disturbances of metabolic regulation in acute intermittent porplyyria and lead poisoning}

The symptoms of acute intermittent porphyria and lead poisoning were compared and found to be similar. In disturbances of porphyrin metabolism there is evidence of enzyme induction or enzyme inhibition. In both cases, however, the clinical diagnosis is based on an increase of metabolites. In lead poisoning, practically every stage of haem synthesis is affected. Too little attention is paid to the increase of uroporphyrinogen observed by BRUGSCH in lead poisoning; the urinary porphobilinogen value remains normal. Uroporphyrin is not formed in the urine during storage.

In both conditions there are disturbances in the mineral and water balance. This is due to an increased secretion of antidiuretic hormone. There is also an increased secretion of melanophoric, somatotropic and thyrotropic hormones by the hypophysis. The increased secretion of hormones by the hypophysis is correlated with anatomical changes in the hypothalamus, which can be demonstrated both in porphyric patients and in experimental animals.

Die praktische Bedeutung und der Wert der Untersuchung des Porphyrinstoffwechsels zeigte sich unter anderem bei der Klärung der Wirkung bestimmter chemischer Stoffe auf die Hämsynthese. Die Forschungen sind einmal auf die Störungen der Hämsynthese selbst gerichtet, zum andern auf die Wirkung dieser Störung auf den Organismus, letzten Endes jedoch auf die Aufklärung der wechselseitigen Wirkungen. Nachdem die Porphyrine erkannt und durch die Forschungen von $\mathrm{H}$. Frscher (1) ihre chemische Struktur geklärt war, wurde in kurzer Zeit auch die Bedeutung der Porphyrinausscheidung bei Gesunden und Kranken erkannt. Durch quantitative und qualitative Untersuchungen hat GüNTHER (2) als erster den Zusammenhang zwischen der Krankheitsform und der pathologischen Porphyrinausscheidung bewiesen; ; dies wurde damals noch mit einer gewissen Zurückhaltung aufgenommen und als besondere Seltenheit angesehen. Heute steht ziemlich fest, daß gewisse klinische Erscheinungen bei der akuten intermittierenden Porphyrie und die Symptome bei Bleivergiftung weitgehend ähnlich sind, doch als Ursache der Symptome der beiden Krankheitsformen weder die Vorstufen noch die Porphyrine selbst bètrachtet werden können. Hingegen ist anzunehmen, daß infolge der gestörten Hämsynthese entstehende Metabolite bzw. die durch diese bedingte biologische Oxydationsstörung eine entscheidende Rolle spielen (3).

\section{Die Hämsynthese beeinflussende Substanzen}

Bald nachdem die physiologische Porphyrinausscheidung erkannt war, richtete sich die Aufmerksamkeit auf die stimulierende Wirkung gewisser Substanzen, was dann zur Klärung der toxischen Porphyrinurien führte (4-12). Es hat sich bei einer ganzen Reihe chemischer Stoffe gezeigt, daß sie die Hämsynthese durch Enzymblockierung stören können (13). Bei gewissen Stoffen ist bei ihrer Wirkung auf die Leberzellen mit Induktion von Enzymen zu rechnen (14). Chemische Substanzen führten auch in Tierversuchen zu vermehrter Porphyrinausscheidung und damit war der Weg zum experimentellen Studium des physiologischen und pathologischen Porphyrinstoffwechsels eröffnet. BRUGSCH (4) hat auf die Wirkung der $\mathrm{Zu}$ sammensetzung der Nahrung auf die Porphyrinausscheidung hingewiesen; dies muß als Erklärung für die, in den Normalwerten der Exkremente gefundenen Unterschiede angesehen werden (15). BRUGSCH (16) stellte weiter fest, da $B$ mit ständig eingehaltener Diät aufgenommene, von ihm als porphyrinagog bezeichnete Substanzen die Porphyrinausscheidung zu steigern vermögen. Im allgemeinen gilt dies für die ätherlöslichen Kopro- bzw. Protoporphyrine.

SHEMin und Mitarbeiter $(17,18)$ hatten durch Isotopenuntersuchungen den Gang der Synthese klargestellt. Dies bildete später die Grundlage zur klinisch-che- 
mischen Diagnose und gewährte zugleich auch tieferen Einblick in die Krankheitsprozesse. Von den experimentellen Porphyrien ausgehend wurden mehrere Verbindungen erkannt, die auf den Pyrrolstoffwechsel schädigend wirken (19). Bei experimentellen hepatischen und erythropoetischen Störungen wurde es durch diese Stoffe möglich, die beiden pathologischen Erscheinungsformen zu trennen. Von den toxisch wirkenden Stoffen können gewisse - wie dies von NAKAO und Mitarbeitern (20) in Zusammenhang mit Griseofulvin beobachtet wurde - auf die Hämsynthese der Leber wie auf die roten Blutkörperchen störend wirken. Auf die hepatische Form hat eigentlich als erster STokvis (5) im Zusammenhang mit Sedormid hingewiesen und später studierten Schmid und Schwartz (21) die Ursache dieser Hämsynthesestörung. Unter bestimmten Bedingungen ist es auch gelungen, den Ort der Blockierung oder der Überproduktion festzustellen. Es steht fest, daß bei der Auslösung akuter Anfälle auch gewisse Medikamente, wie z. B. Barbiturate, Sulfonamide usw., eine wichtige Rolle spielen (19), durch welche man auch zugleich zu der Kenntnis der Natur der Störung der Enzymsynthese gelangte. Heute ist mehr oder weniger sicher, daß diese Stoffe auf die $\delta$-Aminolävulinsäure-Synthetase, - welches sich als ein induzierbares Enzym erwies - Einfluß haben. Die Hemmstoffe der Eiweißsynthese hemmen zugleich auch die Enzymsynthese und vermögen auch die Steigerung in der Bildung der Porphyrine oder ihrer Vorstufen zu verhindern. Auf ähnliche Weise kann eine hemmende Wirkung durch kohlenhydrat- bzw. eiweißreiche Nahrung erreicht werden (22).

Eine besondere Rolle im Zusammenhang mit dem Porphyrinstoffwechsel kommt dem Blei zu, das infolge seiner Bedeutung in der Industrie schon früh die Aufmerksamkeit der Forscher auf sich lenkte. Das Blei unterscheidet sich von anderen, Porphyrinurien und Hämsynthesestörungen verursachenden Stoffen, indem es in der Enzymblockierung eine dominierende Rolle spielt und nahezu auf jeder Stufe der Hämsynthese zur Geltung kommt, wenn auch in den einzelnen Phasen in verschiedenem Grade. Manche Substanzen hingegen sind als induzierende Stoffe in der Enzymsynthese bekannt.

Die Vermehrung und die erhöhte Ausscheidung der Porphyrine bzw. ihrer Vorstufen lassen sich daher in gewissen Fällen auf Enzymüberproduktion, in anderen Fällen auf Hemmung der Enzymbildung bzw. ihrer Wirkung zurückführen.

\section{Störungen der Hämsynthese bei Bleivergiftung}

\section{1. $\delta$-Aminolävulinsäure, Porphobilinogen}

Bei Bleivergiftung zeigen sich bereits in der $\delta$-Aminolävulinsäure ${ }^{1}$ )-Ausscheidung Störungen (23). Es wird angenommen, daß ALS in höherer Konzentration die ALS-Synthetase hemmt. Als Ursache der ALS-Ver-

1) Abkürrungen: $\mathrm{ALS}=\delta$-Aminolävulinsäure, $\mathrm{PBG}=$ Porphobilinogen. mehrung bei Bleivergiftung wird in erster Linie die Hemmung der ALS-Dehydrase angesehen, wie dies die in vitro Versuche von RuBrno (24) zeigen. Diese Hemmung kann an sich Uisache der ALS-Vermehrung sein. In seinen Versuchen wirkte nämlich das Blei auf die von Glycin und Succinyl-CoA ausgehende Synthese weniger hemmend bzw. die Hemmung war nur bei sehr hoher Konzentration ausgesprochen. Bezüglich der Ausscheidung des Porphobilinogens ${ }^{1}$ ) sind die Literaturangaben nicht einheitlich. Bei Bleivergiftung wird das PBG-Niveau im allgemeinen als normal oder leicht erhöht beschrieben. So fanden z. B. HAEger-AronseN (25), Forminje und Lehr (26) bei Kaninchen, RuBino (24), TANABE (27), WaLdENSTRöm (28) und Watson (29) bei Menschen das PBG vermehrt, während HAEGERAronsen (25), Brugsch (4) sowie Kretser und WALDRON (30) bei Bleivergifteten normale Ausscheidung beobachtet haben. Wir selbșt registrierten in unseren Fällen normale Ausscheidung (31), es kamen aber auch extrem niedrige Werte vor, wie auch in den Fällen von Albahary und Mitarbeitern (32).

Bei Bleivergiftung ist die Hemmung der ALS-Dehydrase in vivo und in vitro verschieden, was auf die Löslichkeit des Bleis zurückzuführen sein dürfte, da dieses in den Geweben in Form von schwer löslichen Phosphaten vorliegt und die Enzymhemmung daher hier niemals einen solchen Grad erreichen kann wie in in vitro Experimenten (33). Das Blei allein vermag den Umwandlungsprozeß $\mathrm{PBG} \rightarrow$ Uroporphyrinogen nicht zu hemmen, die Anwesenheit von ALS aber bewirkt auch auf dieser Stufe eine hemmende Wirkung des Bleis, wodurch sich die in der Bildung des Uroporphyrinogens beobachteten Unterschiede erklären ließen.

Die hohe ALS-Ausscheidung ist jedoch keineswegs durch die Enzymhemmung allein zu erklären. Es muß auch an eine gesteigerte ALS-Synthese gedacht werden, die sich unter anderem in der kompensatorischen Hyperfunktion der exythropoetischen Gewebe und der exhöhten Destruktion der roten Blutkörperchen manifestiert, wie dies Rubino, Prato und Fiorina (34) in ihren die Lebensdauer der roten Blutkörperchen betreffenden Untersuchungen mit ${ }^{51} \mathrm{Cr}$-Erythrocyten schon früher nachweisen konnten.

Mit der Klarstellung des Hämsynthesevorgangs erweiterten sich die diagnostischen Möglichkeiten bei Bleischäden und damit wurden auch die Beziehungen der bleibedingten Porphyrinopathien zur Hämsynthese klar. Außer den schon früher bekannten Koproporphyrinurien wurde eine pathologisch gesteigerte ALS Ausscheidung klinisch zuerst von HAEGER (23) beobachtet. Wir selbst (31) haben in unseren Untêrsuchungen im Tagesurin von 53 Bleiarbeitern in über $90 \%$ pathologisch hohe ALS-Ausscheidung gefunden; ebenso war in den Untersuchungen von HAEGERAronsen (25) die Korrelation zwischen ALS und Blei besser verwertbar als die ALS-Korrelation zum Koproporphyrin. Die pathologische ALS-Ausscheidung ist das früheste Symptom der Bleivergiftung. Manche Autoren halten die pathologische Ausscheidung für 
spezifisch $(25,27,30,35-38)$. Nach Heilmeyer (38) steht die ALS-Ausscheidung zur Bleivergiftung in einer viel engeren Beziehung als die Porphyrin- oder auch die Bleiausscheidung. Nach seinen Untersuchungen finden sich die höchsten ALS-Werte bei der Bleivergiftung, doch haben wir auch eine extreme Ausscheidung bei akuter intermittierender Porphyrie beobachten können (150 mg pro Tag). In den Erythrocyten Bleivergifteter nimmt in vitro die PBG-Bildung stark ab, wird jedoch bei Hinzufügung von Cystein normalisiert, was als Beweis dafür gelten kann, daß das Blei mit den SHGruppen des Enzyms reagiert (38). Die praktische Bedeutung der mit dem Stuhl entleerten Porphyrine ist geringer (39).

\section{Uroporphyrinogen, Uroporphyrin}

Eine relativ wenig beachtete Erscheinung ist das Uroporphyrin, besonders dessen farblose Vorstufe; die fallweise stärkere Ausscheidung von Uroporphyrinogen wurde von Brugsch (40) im Jahre 1957 bei akuter Bleivergiftung bei einer Schwangeren beschrieben. BOYET und BUtTER IVORTH (41) und ebenso wir selbst machten öfters die gleiche Beobachtung bei Bleivergiftungen. Von unseren Befunden führen wir hier die quantitativen Verhältnisse von drei Fällen an:

1. Uroporphyrinogen $4600 \mu \mathrm{g} / 24 \mathrm{Stdn}$.; Uroporphyrin $400 \mu \mathrm{g} / 24 \mathrm{Stdn}$.

2. Uroporphyrinogen $3206 \mu \mathrm{g} / 24 \mathrm{Stdn}$; Uroporphyrin $249 \mu \mathrm{g} / 24$ Stdn.

3. Uroporphyrinogen $587 \mu \mathrm{g} / 24 \mathrm{Stdn}$.; Uroporphyrin $68 \mu \mathrm{g} / 24 \mathrm{Stdn}$.

Das Erscheinen großer Mengen Uroporphyrinogen spricht dafür, $\mathrm{da} ß$ sowohl die Oxydation wie die Decarboxylierung des Uroporphyrinogens unvollständig ist. Im Zusammenhang mit Bleivergiftung wies HeIlmeyer (38) auf die Vermehrung des Uroporphyrins und die Störung der Decarboxylierung in den roten Blutkörperchen hin. Die durch das Blei verursachte Membranschädigung ermöglicht das Austreten des Uroporphyrinogens, wodurch es dann zu gesteigerter Ausscheidung kommt. Das ausgeschiedene Uroporphyrinogen konnte keineswegs von der während des Stehens im Urin stattgefundenen PBG $\rightarrow$ Uroporphyrinogen Transformation herstammen, da das PBGNiveau im Harn der oben erwähnten Fälle niedrig, in einigen Fällen sogar kaum nachweisbar war. Nach manchen Befunden ist das Porphyrin bei Bleiexposition auch im Plasma erhöht (41). Die Ursache für das Ansteigen des freien Porphyrins in den Erythrocyten liegt darin, daß in der letzten Phase der Synthese infolge der Blockierung des Goldbergschen Fermentes die Bindung des Eisens an das Protoporphyrin behindert wird (4).

\section{Elektrolyt- und Wasserhaushaltgleichgewichts- Störungen bei Porphyrinopathien}

Die Kenntnis der biochemischen Störungen bei der Bleivergiftung und der akuten intermittierenden Por- phyrie - ALS (23), Koproporphyrin (43), Ausscheidung von Uroporphyrinogen (4) mit dem Harn, von Porphyrinen mit dem Stuhl $(4,39)$, außerdem die Wirkung des Bleies auf den intermediären Stoffwechsel (44) - stellte die Frage des Pathomechanismus der porphyrinopathischen Erscheinungen in der Vordergrund, die in ihrer Gesamtheit schon seit 1952 wiederholt Gegenstand unserer klinischen wie experimentellen Untersuchungen waren (somatotropes und antidiuretisches Hormon, histologische Veränderungen usw. (4553)). Vor allem sei auf jene, bei der akuten intermittierenden Porphyrie wie bei der Bleivergiftung auftretenden Erscheinungen hingewiesen, die in eigenen Arbeiten klargestellt sind und mit deren klinischen Symptomen sich in vergleichenden Untersuchungen auch DAGG und Mitarbeiter (54) befaßt haben.

Zunächst seien die Verschiebungen der Elektrolyte und die Störung des Wasserhaushaltes erwähnt, die bei akuter intermittierender Porphyrie zum erstenmal von Rórt (55) im Jahre 1935 beschrieben worden sind. In einem seiner Fälle bestand während der akuten Phase Hypochlorämie, Hyponaträmie, Oligurie, Wasserretention mit Hyposmolarität des Harnes; in dieser extrarenalen Milieuverschiebung stieg der Reststickstoff auf $280 \mathrm{mg} / 100 \mathrm{ml}$. Nach der akuten. Phase blieb die Hyposmolarität noch wochenlang weiter bestehen und auf Wasserbelastung auch dessen Retention. Dieser ersten Beobachtung folgten mehrere ähnliche anderer Forscher, mit verschiedener Interpretation des Pathomechanismus (56-62).

Eine im wesentlichen ähnliche Erscheinung konnte RóTH $(63,64)$ bei Bleivergiftung beobachten; daraus ließ sich auf einen in gewissem Sinne gemeinsamen Pathomechanismus schließen, dessen Natur aufzuklären damals, im Rahmen der gegebenen Möglichkeiten, nur hypothetisch möglich war. Diese Möglichkeit ergab sich beim hepatorenalen Syndrom mit Hypochlorämie, Oligurie und Azotämie oder ohne diese. In einem Fall von akuter intermittierender Porphyrie von Hellman und Mitarbeitern (61) war außer der bestehenden Hyponaträmie die $\mathrm{Ca}$ - und $\mathrm{Mg}$-Ausscheidung mit dem Harn dermaßen hoch, daß bei dem entsprechend äußerst niedrigem Niveau im Serum, Tetaniesymptome auftraten. BRANDT (65) bringt die Ca-Ausscheidung bei Bleivergiftung mit dem Blei in Verbindung und beschreibt die Ca-Ausscheidung auch auf dem Darmwege als erhöht, was auf eine Parallelität zwischen den beiden Krankheitsbildern - der akuten intermittierenden Porphyrie und der Bleivergiftung - hinsichtlich der Geschehnisse im Ionenmilieu des Organismus hinweist.

Iñ unseren 1952 durchgeführten Untersuchungen (45) standen bei den mit Bleiacetat vergifteten Ratten Veränderungen der Nieren im Vordergrund; die Glomeruli waren groß und hyperämisch, zwischen den Gefäßschlingen und der Boivmanschen Kapsel sammelte sich. Ödem oder fibrinhaltiges Exsudat an, von Fall zu Fall mit roten Blutkörperchen, oder desquamierten Endothelzellen. In den geschädigten Kanälchen war 
minimale fettige Degeneration zu sehen, die Zellen waren geschwollen, das Lumen: eingeengt. BRUGSCH (4) hatte bei Bleivergiftung ebenfalls Schädigung der Nieren feststellen können. BEAWEER (66) fand elektronenmikroskopisch in den Nieren bleivergifteter Ratten im Kern der Epithelzellen der geschädigten proximalen Kanälchen Einschlußkörper, die nicht von Viren herstammen.

Die Veränderungen in der Leber waren im wesentlichen Hyperplasie und Hyperfunktionserscheinungen im Zentrum der Lobuli, in der intermediären Zone und an der Peripherie. Ivanov (67) berichtete über sekundäre Leber-, Nieren- und Lungenveränderungen bei Stọrungen des Porphyrinstoffwechsels.

\section{Hormonale Störungen bei Porphyrinopathien}

Im Hinblick auf die Hämsynthesestörung und auf die Möglichkeit einer veränderten Hormonabsonderung wurden zahllreiche Untersuchungen durchgeführt.

1. Wir konnten durch Injektion von Harn bleivergifteter Kaninchen eine vermehrte Absonderung des Melanophorenhormons an Fröschen nachweisen, jedoch zeigte sich, daß Hinsberg und Rodewald (68) bereits 1938 durch Porphyrinbehandlung das Freiwerden des Melanophorenhormos bei Fröschen nachweisen konnten. Ihrer Meinung nach steht diese Erscheinung unter der Regelung des Hypothalamus.

2. Den Beweis für eine gesteigerte Absonderung des antidiuretischen Hormons erbrachten wir in einer weiteren Versuchsreihe (45, 49). Mit wäßr. Bleiacetatlösung haben wir bei 2,5 bis 3,0 kg schweren Kaninchen akute und subakute Bleivergiftungen herbeigeführt. Mit dem Hypophysenextrakt der bleivergifteten Kaninchen behandelte Tiere schieden von der in den Magen eingeführten Wassermenge signifikant weniger aus als die Kontrolltiere; während der Wasserretention nahm der Eiweißgehalt des Serums ab. Die Erscheinung konnten wir bei 8 Tieren eindeutig nachweisen.

Die vorübergehend gesteigerte Ausscheidung des antidiuretischen Hormons bei akuter intermittierender Porphyrie wurde als Hypothese zum erstenmal in der Mitteilung von Hellman, Tschudy und BartTer (61) erwähnt. Dies durch Beweis zu belegen ist ihnen jedoch nicht gelungen. Experimentell wurde die Hypothese zum erstenmal durch Nielsen und ThorN (69) bewiesen, indem sie bei akuter intermittierender Porphyrie den ausgeschiedenen antidiuretischen Stoff quantitativ bestimmen konnten; auf Vasopressin bezogen betrug dieser das Mehrfache der normalen Menge.

3. Die erhöhte Absonderung des thyreotropen Hormons wurde schon früher von SüMEGI (70) nachgewiesen und durch unsere späteren Untersuchungen erneut bestätigt (49). In unseren akuten und subakuten experimentellen Bleivergiftungen war die Steigerung des thyreotropen Hormons vom histologisch charakteristischen Bild der Schilddrüse begleitet. Die entsprechenden klinischen Symptome zeigten sich in erhöhtem Grundumsatz, kompensierter Alkalose, Abmagerung, erhöhter Adrenalinempfindlichkeit. Frühere Versuche (71) hatten bereits erwiesen, da $\dot{\beta}$ zentrale Hyperthyreose auch während der Porphyrinbehandlung auftritt, etwas weniger ausgeprägt als bei der bleibedingten Porphyrie. Chronische Bleivergiftung kann auch zu metaplastischer Schilddrüsengeschwulst führen (72).

Nach SANSTEAD (73) ist in der Schilddrüse chroniscb bleivergifteter Tiere die ${ }^{131} \mathrm{~J}$-Aufnahme in vitro wie in vivo gehemmt. In gewissen Fällen war die ${ }^{131} \mathrm{~J}$-Aưfnahme auf Darreichung von TSH verstärkt; das TSH der Hypophyse war vermindert, doch betrachtet der Autor diese Erscheinung als sekundär.

Bei akuter intermittierender Porphyrie ist übrigens die Hyperfunktion der Schilddrüse bekannt, ebenso Subfebrilität und Tachykardie, deren Ursache neuerdings auf zentralnervöse Störungen zurückgeführt wird (74). Hypertensive Zustände erwecken bei den sowieso unruhigen Kranken einen hyperthyreotischen Anschein. Bei in üblicher Weise durchgeführten Untersuchungen zeigt sich der Grundumsatz oft erhöht. Ebenso wurde in manchen Fällen auch die an das Serumeiweiß gebundene Jodmenge als erhöht registriert (75). Wir selbst haben im Laufe unserer vielzähligen an Kranken durchgeführten Bestimmungen keine pathologische Steigerung des PBJ und der radioaktiven Jodaufnahme gefunden. Wir konnten zwischen Sauerstoffverbrauch, dem Grad der Jodaufnahme und den PBJ-Werten keine strenge Korrelation finden. Ein erhöhter PBJ-Wert wird übrigens auf die Störung der Leberfunktion zurückgeführt; nach SANSTEAD (73) kann das Blei im Eiweiß an die Stelle des Jods treten.

4. Die diabetogene Wirkung des somatotropen Hormons konnten wir in unseren Tierversuchen bestätigen. Mit Hypophysenextrakten bleivergifteter Kaninchen wurden Ratten injiziert; der Ablauf der Glucosebelastungskurve bei den Versuchstieren zeigte den diabetischen Typ (45).

Perlroth, Tschudy, Waxmann und Odeli (76) wiesen auch auf die Veränderung der Wachstumshormonabgabe hin. Bei akuter Porphyrie reagieren die Kranken auf Glucosebelastung mit paradoxer $\mathrm{Zu}-$ nahme des Hormonspiegels.

\section{Veränderungen des zentralen Nervensystems bei Porphyrinopathien}

In unseren an bleivergifteten Kaninchen durchgeführten Versuchen (49) sahen wir im Zentralnervensystem makroskopisch im Wood-Licht rötliche Fluoreszenz in der weißen Substanz des Hirns und des Rückenmarks, ebenso in der weichen Hirnhaut und darunter. Histologisch war an der Grenze der grauen und weißen Substanz des Hypothalamus, subependymal rund um die Ventrikel, häufig im Rückenmark und in den interspinalen Ganglien berétts 24 Stunden nach dem Einsetzen der Intoxikation reaktive herdförmige Glia- 

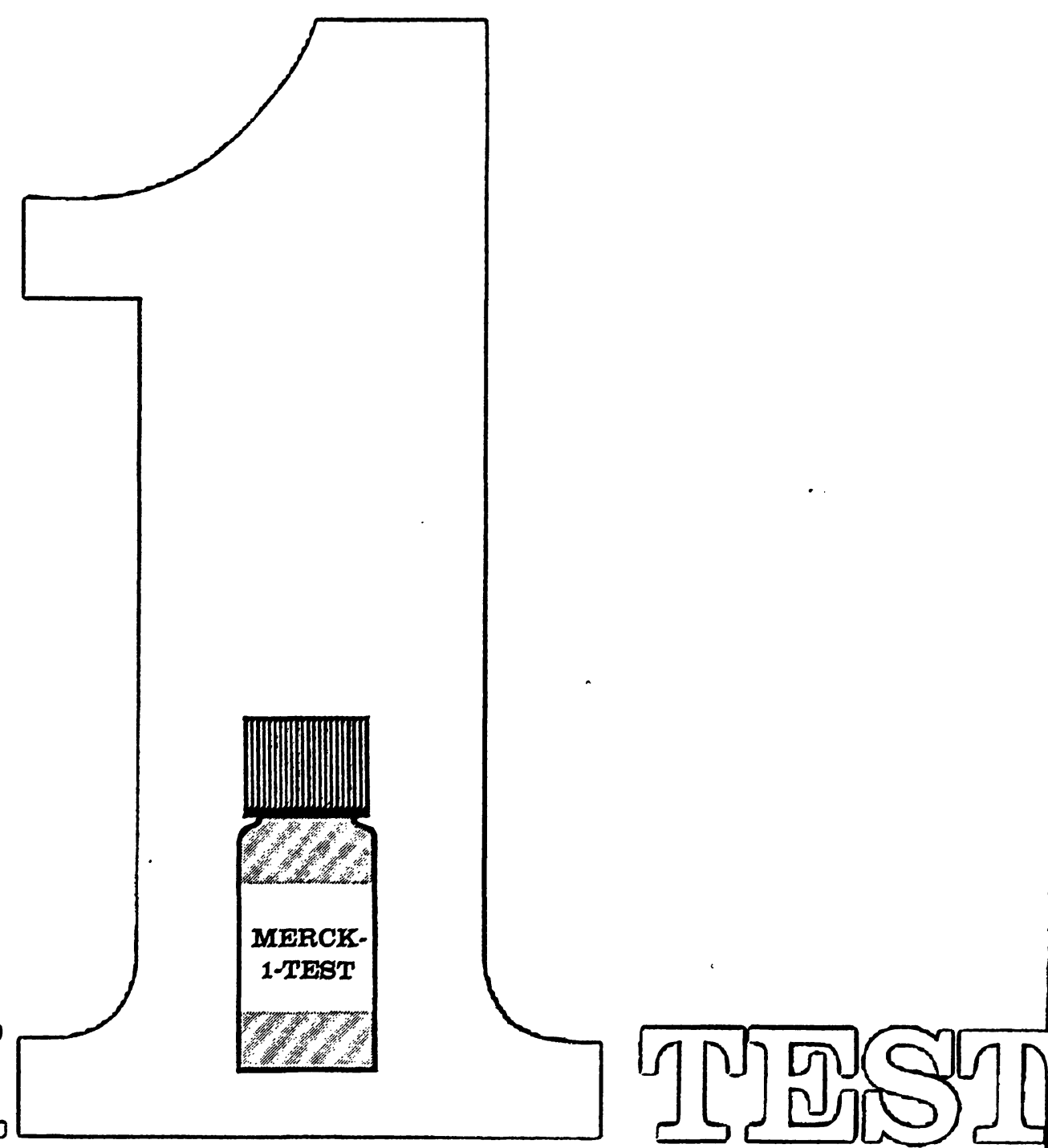

Ein Test in einem Glas

Cholinesterase

GOT (UV)

GPT (UV)

HBDH (UV)

LDH (UV)

Fine neue Konzeption für Sicherheit

und Zeitersparnis in Klinik- und

Praxislabor

Öffnung der 1-Test-Gläser

im Handumdrehen

Lösungsmittel in der Packung

enthalten; daher kein destilliertes

Wasser notwendig

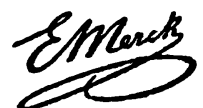

E. MERCK AG - DARMSTADT 
AUTOMATION MED. LABORATORIUMSUNTERSUCHUNGEN

speziell auf den Gebieten der klinischen Chemie, Haematologie, Serologie, Protein- und Hormonchemie.

Wir suchen möglichst ab 1. Juli 1969 oder später einen

\section{ARZT}

mit Kenntnissen in der Laboratoriumsmedizin, die einem internationalen Standard entsprechen.

Wir erwartenieine kontaktreiche und reisefreudige Persönlichkeit mit großem Einfühlungsvermögen in die Belange der klinischen Forschung und weit vorausblickendem Planungsvermögen für praktikable Möglichkeiten der Automation im Laboratorium.

Die Aufgabe des erfolgreichen Kandidaten wird sein, den weltweiten Verkauf von klinischen Geräten zu fördern und die Firma in allen verkaufs- und expansionspolitischen Fragen zu beraten.

Voraussetzung ist Beherrschung der englischen Sprache in Wort und Schrift. Französische Sprachkenntnisse sind erwünscht.

Wir bieten unter Gewährung einer ausreichenden Einarbeitungszeit in die speziellen Probleme der Firma mit Training im amerikanischen Stammhaus und den europäischen Niederlassungen die

\section{LEITUNG DER KLINISCHEN ABTEILUNG IN DER EUROPAISCHEN ZENTRALE}

mit Hauptsitz in Genf. Diese Abteilung bearbeitet sämtliche Länder außerhalb Nordamerika.

Diese Position entspricht einer Daverstellung mit hoher Dotierung und zusätzlicher Spesen- und Aufwandsentschädigung. Altersversorgung.

Chiffre KC 4/69 118 Merkur Werbung, 5213 Spich/Bez. Köln, Merkur-Haus, Hauptstr. 23-27

\section{Magenoperation und}

\section{Magenoperierter}

\section{Herausgegeben von}

Prof. Dr. med. Heinrich Bartelheimer

Direktor der I. Medizinischen Universitäts-Klinik Hamburg

\section{Prof. Dr. med. Hans-Joachim Maurer}

Institut und Klinik für Medizinische Strahlenkunde der Universität Düsseldorf

Prof. Dr. med. Hans W. Schreiber

Chefarzt der Chirurgischen Abteilung des Marien-Krankenhauses, Hamburg

unter Mitwirkung von

Priv.-Doz. Dr. med. Kurt Müller-Wieland

Oberarzt der I. Medizinischen Universitäts-Klinik Hamburg

Groß-Oktav. XVI, 489 Seiten. Mit 210 Abbildungen und

2 mehrfarbigen Tafeln. 1969. Ganzleinen DM 88,-

Durch die Zusammenarbeit verschiedener Disziplinen und durch die Entwicklung neuer Methoden sind in der Gastrologie neue Erkenntnisse über Form und Funktion des gesunden und kranken Magens gewonnen worden. Die chirurgischen Leistungen wurden durch die Einbeziehung internistischer, röntgenologischer, nuklearmedizinischer und vor allen Dingen biochemischer Verfabren ergänzt. Gastroenterologen, Chirurgen, Röntgenologen, Hämatologen, Osteologen und Pädiater sowie Vertreter anderer Fächer haben aus diesen Gründen hier die Probleme der Magenoperation und der Magenoperierten dargestellt. Erst langjährige und vergleichende Erfahrungen gestatten eine kritische Bewertung, wie sie jetzt hier vorgelegt wird.

Das Buch wendet sich an alle Disziplinen der Medizin.

Verbindliche und eindeutige Aussagen zur Diagnostik und zum therapeutischen Vorgehen finden der praktizierende Arzt, det die erste Indikation zu stellen hat, ebenso wie Gastroenterologen, Internisten, Chirurgen und Röntgenologen.

\section{Walter de Gruyter \& Co - Berlin}


vermehrung $z u$ sehen. Es war hauptsächlich an der Berührungslinie der grauen und weißen Substanz zu beobachten, also an der Stelle, an der bei dem Vergiftungsprozeß das Porphyrin zum erstenmal erscheint und sich anhäuft. Später bilden sich an der Stelle der reaktiven Gliose Demyelinisierungsherde und an den gleichen Stellen finden sich auch Gefäßveränderungen. Das Endothel der Kapillaren ist hier geschwollen, ringsherum zeigen sich winzige Blutungen. Nach einigen Tagen war Mobilisierung der Makrophagen, Erscheinen von siderophoren Zellen zu beachten und in der vermehrten Oligodendroglia erscheinen Lymphocyten. In den Ganglienzellen kommt es bereits nach 24 Stunden zu degenerativen Erscheinungen in Form von exzentrischer Lagerung, schlecht und allmählich überhaupt nicht mehr anfärbbarer Kerne, peripherer Anhäufung chromatophiler Substanzen. Auffallend sind die nekrobiotischen Veränderungen der interspinalen Ganglienzellen in Form einer fibrinoiden Nekrose. Nach Brandr (65) lagert sich das Blei besonders im Thalamus ab.

Den Untersuchungen von PerLroth und Mitarbeitern (77) über die Veränderungen in den supraoptischen und paraventrikulären Kernen kommt deshalb eine hohe Bedeutung $z u$, weil in Fällen der akuten intermittie- renden Porphyrie, in denen Elektrolytverschiebung fehlte, dieser Bereich sich histologisch intakt zeigte, wogegen in den oben beschriebenen Fällen mit Elektrolytverschiebungen bei der Sektion im Hypothalamus akute und chronische Malazie vorgefunden wurde, von der ausschließlich die Eminentia mediana und der Hypothalamus-Hypophysentrakt betroffen waren. Auf dem Gebiet der Läsion waren die Ganglien fokal geschwollen und wiesen Fragmentationen auf mit umschriebener Demyelinisierung.

Besonders hervorzuheben ist die beschriebene Hypothalamusläsion mit Hinblick darauf, daß sie genau der anatomischen Lokalisation entsprach, welche für die Vasopressinbildung und -abgabe in Frage kommt (78 bis 80 ).

Spatz und Mitarbeiter (81) fanden bei bleivergifteten Katzen in den Hirnganglienzellen degenerative Erscheinungen von leichteren Zellveränderungen bis zum Plasmazerfall bzw. bis zur Kolliquation der Zellen und amöboider Umwandlung der Glia. Nach Villawerde (82) handelt es sich hier um eine direkte Wirkung des Bleies auf das Parenchym des Nervengewebes und nicht um eine indirekte, seitens der Gefäße und der Bindegewebe.

\section{Literatur}

1. Fischer, H. und H. Orth, Die Chemie des Pyrrols. Akad. Verlags-Ges. Leipzig (1937). - 2. GüNTHER, H., Dtsch. Arch. Klin. Med. 105, 89 (1912). - 3. LABBE, R. F., Lancet, London 1967/I, 1361. - 4. Brugsch, J., Porphyrine. 2. Aufl. J. A. Barth, Leipzig (1959). - 5. Stokvis, B. J., Zschr. klin. Med. 28,1 (1895). 6. Schmid, R. und S. Scriwartz, Proc. Soc. Exp. Biol. Med. 81 685 (1952). - 7. Goldberg, A. und C. Rimington, Proc. Roy. Soc. B. 143, 257 (1955). - 8. Ockner, R. K. und R. Schmid, Nature, London 189, 499 (1961). - 9. DE Matreis, F. und B. E Prior, Biochem. J. 83, 1 (1962). - 10. DE Matreis, F. und C. Rimington, Brit. J. Derm. 75, 91 (1963). - 11. Rimington, C. und G. Ziegler, Biochem. Phamacol. 12, 1387 (1963). - 12. Prato, V., U. Mazza und A. Ramello, Lancet, London 1964/I, 1276. - 13. GajDOS, J. A., M. GajDos-TöröK und I. H. BÉNARD, Porphyries. Etude chimique et biologique. Paris (1958). - 14 Granick, S., J. biol. Chemistry, 24, 1359 (1960). - 15. GoreCZKY, L., I. Róth und M. BRECKNER, diese Z. 6, 489 (1968). - 16. Brugsch, J. Zschr. inn. Med., Leipzig, 13, 781 (1958). - 17. Shemin, D. und J. Wirtenkerg, J. biol. Chemistry 192, 315 (1951), - 18. Shemin, D. und S. Kumin, J. biol. Chemistry 198, 827 (1952). - 19. Granick, S., J. Amer. Med. Ass. 190 475 (1964). - 20. NaKao, K., O. Wada, F. Takaku, S. Sassa, Y. Yano und G. Urata, J. Laborat. Clin. Med., S. Louis 70, 923 (1967). - 21. Sснмid, R., in The Metabolic Basis of Inherited Disease (edited by J. B. Stanbury, S. B. Wyngarden und D. S. Frederickson) New York (1966). - 22. Rose, J. A., E. S. HellMAN und D. P. Tschudy, Metabolism, Baltimore 10, 514 (1961). 23. HAEGER, B., Scand. J. Clin. Laborat. Invest. 9, 211 (1957). 24. Rubino, G. F., Panminerva Med. 4, 340 (1962). - 25. HaegerAronsen, B., Scand. J. Clin. Laborat. Invest. 12, Suppl. 47 (1960). 26. ForminJe, P. und C.. F. G. Lehr, zit. Haeger-Aronsen (25). 27. Tanabe, Y., Jap. J. Nation's Health 28, 386 (1959). - 28. Waldenström, J., Acta Med. Scand. Suppl. 82 (1937). - 29. Watson, C. J., V. Hawkinson und I. Bossenmaier, Transact. Ass. Amer. Physicians 66, 144 (1953). - 30. DE KRETser, A. J. und H. A. WaLdRon, Brit. J. industr. Med. 20,35 (1963). - 31. Róth, I. und L. GoreczKy, Zschr. inn. Med., Leipzig 16, 1078
(1961). - 32. Albahary, C., R. Truhaut, C. Boudene und H. Desollle, Presse Méd. Paris 69, 2121 (1961). - 33. Goldberg, A., H. Aschenbrucker, G. E. Cartwright und M. M. Wintrobe, Blood 11, 821 (1956). - 34. Rubino, G. F., V. Prato und L. Fiorina, Folia Med. Napoli 42, 1 (1959). - 35. GatTner, H. und G. Schrantz, Dtsch. med. Wschr. 89, 1027 (1964). - 36. GrigGs, R. C. und J. W. Harris, Clin. Res. 6, 188 (1958). - 37. TischKoff, G. H., B. Granville, R. Rosen und W. Dameshek, Acta Haematol. (Basel) 19, 321 (1958). - 38. Heilmeyer, L., Die Störungen der Bluthämsynthese. 1. Aufl. G. Thieme, Stuttgart (1964). - 39. Haeger-Aronsen, B., Scand. J. Clin. Laborat. Invest. 14, 397 (1962). - 40. BRugșCH, J., Zschr. inn. Med., Leipzig 12, 704 (1957). - 41. Boyetr, J. D. und C. E. Butterworth, Amer. J. Med. 32, 884 (1962). - 42. WALDRON, H. A., Brit. J. Industr. Med. 21, 315 (1964). - 43. FRANKE, K. und S. LITZNER, Zschr. klin. Med. 178, 2 (1935). - 44. BAIER, H., K. H. BäsSLER und S. LrTzNER, Naunyn-Schmiedebergs Arch. exper. Path. 229, 495 (1956). - 45. RótH, I., I. SÜMEGI und L. GORECZKY, Acta Morphol. Hung. II, 423 (1952). - 46. GoreczKY, L., I. Róth, I. Sümegi und Gy. VAjDA, Acta Med. Hung. VI. Suppl. 1, 93 (1954). - 47. Róth, I., L. GoreczKY, J. Molnír und I. SÜMEGI, Orv. Hétil. Budapest 97, 767 (1955). - 48. SÜMEGI, I., L. GoreczKy und I. Rótr, Kisérl. Orvostudom. Budapest 8 , 428 (1956). - 49. SüMEgI, I., L. GoreCzKY und I. Róth, Acta Morphol. Hung. VII, 183 (1956). - 50. RóTH, I., L. GoRECZKY, J. Molnár und I. Sümegr, Zschr. inn. Med. Leipzig 12, 707 (1957). - 51. GoRECZKY, L. und I. Róth, Kisérl. Orvostudom. Budapest 14, 468 (1962). - 52. GoreCzKY, L. und I. Róth, MÁV Eü. Int. Tud. Közl. IV,49 (1962). - 53. Róth, I. und L. GoreCZKY, MÁV Eü. Int. Tud. Közl. IV, 197 (1962). - 54. DAGG, J. H., A. Goldberg, A. Lochhead und J. A. Smith, Quart. J. Med. $X X X I V, 134$ (1965). - 55. Rórr, I., Dtsch. Arch. klin. Med. 178, 2 (1935). - 56. LindeR, G. C., Lancet, London 1947/II, 649. - 57. Abrahams, A., C. J. Gavey und N. F. Mclagan, Brit. Med. J. 1947/II, 327. - 58. Davres, D., Brit. Med. J. 1949/I, 846. - 59. Prunty, F. T. G., J. Clin. Invest. 18, 690 (1949). 60. Edelman, I. S., J. Leibman, M. P. O'Meara und L. IV. 
Birkenfeld, J. Clin. Invest. 37, 1236 (1958). - 61. Hellman, E. S., D. P. Tschudy und F. C. Bartrer, Amer. J. Med. 32, 734 (1962). - 62. Ludwig, G. D. und M. Goldberg, Ann. N. Y. Acad. Sci. 104, 710 (1963). - 63. RórH, I., Orv. Hétil. Budapest 81, 38 (1937). - 64. Rótr, I., Orv. Hétil. Budapest 82, 3 (1938). 65. BRANDT, H. H., Zschr. inn. Med. Leipzig 12, 7 (1957). 66. Beawer, D. L., Amer. J. Path. 39, 195 (1961). - 67. Ivanow, E. D., Dtsch. med. Wschr. 110, 1783 (1968). - 68. HinsBerg, K. und W. Rodewald, Naunyn-Schmiedebergs Arch. exper. Path. 191, 1 (1938). - 69. Nielsen, B. und A. Thorn, Amer. J. Med. 38, 345 (1965). - 70. SüMEgr, I., Acta Morphol. Hung. I, 459 (1951). - 71. Purnoky, Gy. und I. SüMEgr, Arch Gewerbepath. Berlin 9, 566 (1939). - 72. ZawirsKa, B. und K. MEdras, Zbl. allg. Path. 111, 1 (1968). - 73. Sanstead, H. H., Proc. Soc. Biol. Med. 124, 18 (1967). - 74. Ridley, A., R. Hierons und J. B. Cavannagh, Lancet, London 1968/II, 708. - 75. Tschudy, D. P., J. Amer. Med. Ass. 191, 114 (1965). - 76. Perlroth, M. G., D. P. Tschudy, A. Waxman und W. D. Odell, Metabolism Baltimote 16, 87 (1967). - 77. Perlroth, M. G., D. P. Tschudy, H. S. Marver, C. W. Berard, R. F. Zetget, M. Rechcigl und A. Collins, Amer. J. Med. 41, 149 (1966). - 78. NesBrt, S. und C. Watkins, Amer. J. Med. Sci. 203, 44 (1942). - 79. Golden, L., Amer. J. Med. Sci. 206, 474 (1943). - 80. NesBit, S., J. Amer. Med. Ass. 124, 286 (1942). - 81. Spatz und Mitarbeiter, zit. Brandt (65). - 82. VILLAWERDE, zit. Brandt (65).

Doz. Dr. med. habil. L. Goreczky MAV-Krankenhaus

Budapest VI

Rudas L. u. 111., Ungarn 